\title{
Levels of serum fibronectin as a biomarker in gastric cancer patients: Correlation with clinical diagnosis and outcome
}

\author{
FARUK TAS, ELIF BILGIN, SENEM KARABULUT, DIDEM TASTEKIN and DERYADURANYILDIZ \\ Institute of Oncology, Istanbul University, Capa, Istanbul 34390, Turkey
}

Received August 27, 2015; Accepted January 28, 2016

DOI: $10.3892 / \operatorname{mco} .2016 .759$

\begin{abstract}
Fibronectin is an important component of the extracellular matrix and serves a role in the pathogenesis of multiple malignancies. The expression of fibronectin also affects the outcome for patients with cancer. The objective of the present study was to determine the clinical significance of the serum fibronectin levels in patients with gastric cancer. A total of 63 patients with a pathologically confirmed diagnosis of gastric cancer were enrolled into the present study. Serum fibronectin concentrations were determined by the solid-phase sandwich enzyme-linked immunosorbent assay method. Age and sex matched healthy controls $(n=30)$ were included in the analysis. The median age at diagnosis was 62 years (range 28-82 years). The baseline serum fibronectin levels of the patients with gastric cancer were significantly higher compared with those in the control group (median values, 606, vs. $193 \mathrm{ng} / \mathrm{ml}$; $\mathrm{P}<0.001$ ). The known clinical variables, including the age of the patient, gender, site of lesion, histology, histological grade, stage of disease and serum tumor markers, including lactate dehydrogenase, carcinoembryonic antigen and cancer antigen 19.9, were not found to be correlated with serum fibronectin concentrations $(\mathrm{P}>0.05)$. In addition, no correlation was observed in serum fibronectin level and response to chemotherapy $(\mathrm{P}=0.12)$. Serum fibronectin concentration demonstrated no prognostic role on survival $(\mathrm{P}=0.43)$. In conclusion, the serum levels of fibronectin may have a good diagnostic marker in patients with gastric cancer. However, its predictive and prognostic values remain to be elucidated.
\end{abstract}

\section{Introduction}

Gastric cancer displays multifactorial etiology and its genetic and immunological background remains to be fully elucidated. It is a malignancy characterized by a highly invasive and metastatic nature, properties which require interaction of

Correspondence to: Dr Faruk Tas, Institute of Oncology, Istanbul University, 118 Millet Street, Capa, Istanbul 34390, Turkey

E-mail: faruktas2002@yahoo.com

Key words: serum, fibronectin, gastric cancer the gastric cancer cells with the extracellular matrix (ECM) through cell adhesion, migration, proliferation and metastasis.

Fibronectin is one of the major structural components of the basement membrane (1-8). Fibronectin serves a prominent role in cell adhesion, growth, migration and differentiation, and it is important for processes, including wound healing and embryonic development via integrins and other cell surface receptors (1-8). Altered expression of fibronectin, degradation and organization have been associated with a number of pathologies, including cancer (1-8). Several of the morphological changes observed in tumor types and tumor-derived cell lines have been attributed to decreased expression of fibronectin, increased fibronectin degradation, and/or decreased expression of fibronectin-binding receptors, including $\alpha 5 \beta 1$ integrins (1-8). Fibronectin is also a candidate biomarker for several solid tumor types, including ovarian, prostate and breast cancer $(1,2)$. In addition to its implication in cancer development, fibronectin also acts as a potential biomarker for treatment-associated resistance (5).

However, a clinical association between the expression of fibronectin and carcinogenesis in gastric cancer was observed in only a few trials (6-8). Increased expression of fibronectin was also detected in gastric cancer cell lines with increased gastric cancer cell proliferation and metastatic potential $(6,7)$. Therefore, an unsatisfactory understanding of the molecular function of fibronectin exists and the possible clinical significance of fibronectin has remained unclear in patients with gastric cancer.

Although all available findings were provided from pre-clinical tissue or cell-based trials, so far, no clinical study has investigated the clinical significance of this ECM marker in the plasma and/or serum in patients with gastric cancer. Therefore, the significance of the serological levels of fibronectin in gastric cancer patients remains unknown. The present study evaluated the soluble serum levels of fibronectin in gastric cancer patients and assessed its associations with the prognosis, known various clinical variables and response to chemotherapy, in order to examine whether fibronectin is a potential novel biomarker for use in the treatment of gastric cancer.

\section{Materials and methods}

Patients and therapy. The present study included 63 consecutive patients admitted to Institute of Oncology, Istanbul University (Istanbul, Turkey). All patients had histologically 
Table I. Patient and disease characteristics.

\begin{tabular}{ll}
\hline Variables & $\mathrm{n}(\%)$ \\
\hline
\end{tabular}

\section{No. of patients}

$63(100)$

Age, years

$\geq 60$

$<60$

$28(44)$

Gender

Male

Female

$38(60)$

Site of tumor

Cardia

Antrum

Undetermined

Histology

Adenocarcinoma

Signet-ring cell

Grade

I-II

III

Undetermined

Tumor stage

1-3

4

Unknown

No. of lymph node involvement

0-2

$\geq 3$

Unknown

Stage of disease

Non-metastatic

Metastatic

Liver metastasis ${ }^{\mathrm{a}}$

Yes

No

Curative surgery ${ }^{\mathrm{b}}$

Yes

No

Unknown

Serum hemoglobin level

Low $(<12 \mathrm{~g} / \mathrm{dl})$

Normal $(\geq 12 \mathrm{~g} / \mathrm{dl})$

Serum leukocyte count

Normal $(<10,000)$

Elevated $(\geq 10,000)$

Serum platelet count

Normal $(<350,000)$

Elevated $(>350,000)$

Serum lactate dehydrogenase level

Normal $(<450 \mathrm{U} / \mathrm{l})$

Elevated ( $\geq 450 \mathrm{U} / \mathrm{l})$

Unknown

$10(16)$

$44(70)$
$14(22)$

22 (35)

27 (43)

12 (19)

13 (21)

38 (60)

$32(51)$

31 (49)

14 (45)

17 (55)

17 (53)

9 (28)

6 (19)

35 (56)

28 (44)

52 (83)

11 (17)

54 (86)

9 (14)

$43(68)$

10 (16)

10 (16)
Table I. Continued.

Variables $\mathrm{n}(\%)$

Erythrocyte sedimentation rate, $/ \mathrm{h}$

Elevated $(\geq 50)$

$16(25)$

Normal $(<50)$

$10(16)$

Unknown

Serum carcinoembryonic antigen level

Normal $(<10 \mathrm{ng} / \mathrm{ml})$

$44(70)$

Elevated $(\geq 10 \mathrm{ng} / \mathrm{ml})$

Unknown

Serum cancer antigen 19.9 level

Normal $(<40 \mathrm{IU} / \mathrm{ml})$

$32(51)$

Elevated ( $\geq 40 \mathrm{IU} / \mathrm{ml})$

Unknown

Response to chemotherapy ${ }^{\mathrm{a}}$

Responsive

Non-responsive

Last status

Alive

$28(44)$

Deceased

$35(56)$

In a metastatic and ${ }^{\mathrm{b}}$ non-metastatic patients.

confirmed gastric cancer and had received no chemotherapy or chemoradiation in the last 6 months. The staging was determined, according to the American Joint Committee on Cancer and International Union against Cancer staging systems. The pre-treatment evaluation included assessment of detailed clinical history and physical examination, with a series of biochemistry tests, including lactate dehydrogenase (LDH), complete blood cell counts, including thrombocytes (PLTs), leukocytes (WBCs), hemoglobin $(\mathrm{Hb})$ and serum tumor markers, carcinoembryonic antigen (CEA) and cancer antigen (CA) 19.9. Those with Eastern Cooperative Oncology Group performance status of $\leq 2$, and appropriate blood chemistry tests received chemotherapy on an outpatient basis, which included different combinations of fluorouracil, folinic acid, capecitabine, docetaxel, cisplatin, epirubicin, with/without radiotherapy depending on the stage of disease. Follow-up programs included clinical, laboratory and radiological assessments performed at 8 -week intervals during chemotherapy or every 12 weeks for no anticancer treatment. The response to treatment was determined according to the revised RECIST criteria version 1.1 (9).

For comparison of serum levels of fibronectin, 30 age- and sex-matched healthy controls were included in the analysis. Informed consent was obtained from all patients and the study was reviewed and approved by the local ethical committee.

Measurement of serum fibronectin levels. Serum samples were obtained on first admission, prior to administration of any adjuvant and metastatic treatment, or follow-up patients. Blood samples were obtained from patients with gastric cancer and healthy controls by venipuncture and clotted at room temperature. The sera were collected following centrifugation 
Table II. Serum fibronectin levels in gastric cancer patients and healthy controls.

\begin{tabular}{lcccccr}
\hline & \multicolumn{2}{c}{ Patients $(\mathrm{n}=63)$} & & \multicolumn{2}{c}{ Controls $(\mathrm{n}=30)$} & \\
\cline { 2 - 4 } Marker & Median & Range & & Median & Range & P-value \\
\hline Fibronectin $(\mathrm{ng} / \mathrm{ml})$ & 606 & $44-950$ & & 193 & $58-614$ & $<0.001$ \\
\hline
\end{tabular}

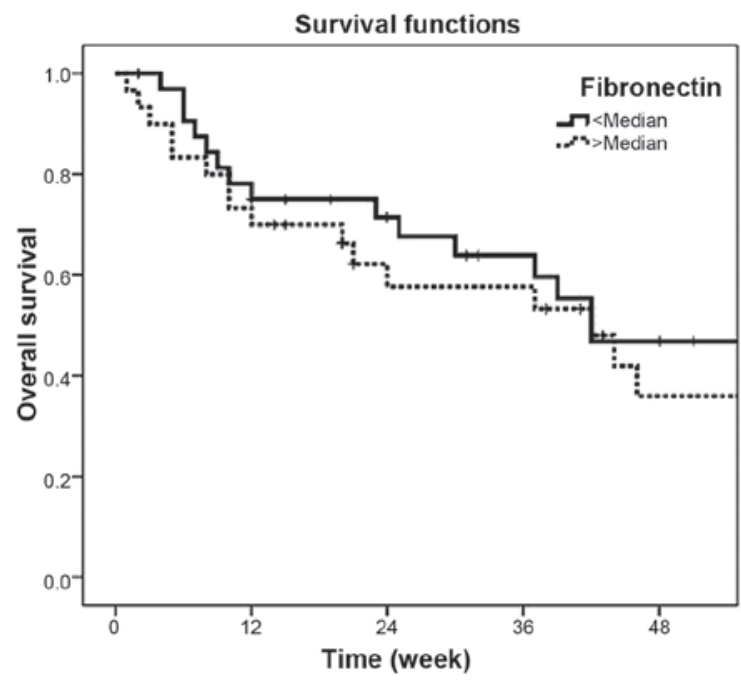

Figure 1. Overall survival curves in gastric cancer patients, according to serum fibronectin levels $(\mathrm{P}=0.43)$.

for $10 \mathrm{~min}$ at $4,000 \mathrm{rpm}$ at room temperature and frozen immediately at $-20^{\circ} \mathrm{C}$ until analysis.

The Fibronectin enzyme-linked immunosorbent assay (ELISA; eBioscience, Vienna, Austria) uses a double-antibody sandwich ELISA to determine the level of human fibronectin in the samples. The serum samples, standards and biotin-conjugate were added to the wells, which were pre-coated with human fibronectin monoclonal antibody and allowed to incubate for $2 \mathrm{~h}$ at $23^{\circ} \mathrm{C}$. Unbound material was washed away. Fibronectin combined with streptavidin-horseradish peroxidase (eBioscience, Vienna, Austria) were added to form an immune complex and were subsequently allowed to incubate for $1 \mathrm{~h}$ at $23^{\circ} \mathrm{C}$. Any unbound material was washed away. Chromogen solution (eBioscience) was added and incubated for $\sim 10 \mathrm{~min}$ in the dark for the conversion of the colorless solution to a blue solution, the intensity of which was proportional to the quantity of fibronectin in the sample. Following the addition of the $100 \mu \mathrm{l}$ acidic stop solution (eBioscience), the color turned yellow. The intensity of the colored reaction product was measured using an automated ELISA reader (RT-1904C Chemistry Analyzer; Rayto, Atlanta, GA, USA) at $450 \mathrm{~nm}$. The results were expressed as $\mathrm{ng} / \mathrm{ml}$.

Statistical analysis. Continuous variables were categorized using median values as cut-off point. Assessment of associations, comparisons between various clinical/laboratory parameters and serum levels of fibronectin assay were accomplished using Mann-Whitney U test. Survival was calculated from the date of first admission to the hospital until mortality resulting from any cause or until the last contact with the patient or any family member. The Kaplan-Meier method was used for estimation of survival of the patient and differences in survival were assessed using the log-rank statistic. $\mathrm{P} \leq 0.05$ was considered to indicate a statistically significant difference. Statistical analysis was performed using SPSS 16.0 software (SPSS, Inc., Chicago, IL, USA).

\section{Results}

A total of 63 patients diagnosed with gastric cancer were enrolled in the present study. The baseline histopathological characteristics and the demographic characteristics of the patients are listed in Table I. The median age at diagnosis was 62 years (range, 28-82 years).

The baseline serum fibronectin levels of the patients with gastric cancer were significantly higher compared with those in the control group (median values, 606, vs. $193 \mathrm{ng} / \mathrm{ml}$; $\mathrm{P}<0.001$; Table II). The known clinical variables, including the age of the patient, gender, site of lesion, histology, histological grade, stage of disease and serum levels of LDH, CEA and CA 19.9 were not correlated with serum fibronectin concentrations ( $\mathrm{P}>0.05$; Table III). Additionally, no association was observed between serum fibronectin level and the response to chemotherapy $(\mathrm{P}=0.12)$.

The median follow-up time was 25 weeks (range, 1-164 weeks). At the end of the observation period, 35 patients (55.6\%) had succumbed to mortality. The median survival time for all patients was $42.0 \pm 4.2$ weeks $(95 \%$ confidence intervals $=33.8-50.2)$. The 1-year survival rates were $42.2 \%$ (95\% confidence intervals $=28.3-56.1)$. The presence of metastasis $(\mathrm{P}=0.03)$, antrum localization $(\mathrm{P}=0.04)$, elevated erythrocyte sedimentation rate $(\mathrm{P}=0.02)$, higher serum CEA levels $(\mathrm{P}=0.01)$, elevated serum CA 19.9 concentrations $(\mathrm{P}=0.04)$ and unresponsiveness to chemotherapy $(\mathrm{P}=0.05)$ exhibited statistically significant worsened survival variables (Table III). However, serum fibronectin concentrations was not associated with prognosis of the outcome $(\mathrm{P}=0.43$; Table III; Fig. 1).

\section{Discussion}

Although overexpression of the fibronectin gene was observed in human gastric cancer, its possible clinical significance has remained unclear in patients with gastric cancer. The possible reason for this unsatisfactory situation is limited data, as only a few studies have been previously performed (6-8).

Performing an immunohistochemical study of the basement membrane antigens, laminin, collagen IV, fibronectin, and type IV collagenase in a series of 87 gastric cancer types, $90 \%$ of the cancers expressed fibronectin, predominantly in the connective tissue at the invading edge of the tumor (6). 
Table III. Distribution and survival comparisons of serum fibronectin levels on various clinical parameters in patients with gastric cancer.

\begin{tabular}{lcc}
\hline & Distribution & Survival \\
Parameter & P-value & P-value \\
\hline
\end{tabular}

Age of patient

$<60 / \geq 60$ years

0.26

Gender

Male/female

0.84

Site of tumor

Cardia/antrum

Histology

Adenocarcinoma/signet ring

Grade

I-II/III

Tumor stage

$1-3 / 4$

No. of lymph node involvement

$0-2 / \geq 3$

Curative surgery

Yes/no

Metastasis

Yes/no

Liver metastasis

Yes/no

Serum hemoglobin level

Low/normal

Serum leukocyte count

High/normal

Serum platelet count

High/normal

Erythrocyte sedimentation rate

High/normal

Serum lactate dehydrogenase level

$$
\text { High/normal }
$$

Serum carcinoembryonic

antigen level

High/normal

Serum cancer antigen 19.9 level

High/normal

Response to chemotherapy

Yes/no

Serum fibronectin level

$</ \geq$ median

Fibronectin expression was also significantly associated with the expanding growth pattern of the neoplasm. However, expression of fibronectin was not significantly correlated with the DNA content or aneuploidy. Similarly, Grigioni et al (7) observed a stronger staining for fibronectin in the connective tissue at the invading edge of the gastric cancer. These findings may reflect compacting of fibronectin pre-existing in the host connective tissue, which may facilitate cancer cell adhesion.

Numerous studies have investigated fibronectin/integrin-mediated signal transduction, and the majority of these have specifically investigated the interaction between fibronectin and integrin through the attachment of cells to the fibronectin-containing ECM (8). However, a large portion of fibronectin is soluble within the body. Soluble fibronectin exists predominantly in the blood plasma and reaches each part of the body through the bloodstream. Similar to tissue, soluble fibronectin also binds integrin and activates consequent signaling events (8).

All previous findings regarding this ECM marker were provided by tissue-cell scale trials. With the exception of the present study, so far, unfortunately, fibronectin was not studied in sera of gastric cancer patients. The present study aimed to determine the clinical significance of the serum fibronectin levels in patients with gastric cancer. Serum levels of fibronectin were quantitatively analyzed by solid-phase ELISA. The results demonstrated that the analysis of serum fibronectin was able to discriminate between the gastric cancer patients and healthy individuals, indicating that fibronectin is a good serological diagnostic marker of gastric cancer. However, no significant associations were observed between the levels of serum fibronectin and the tumor characteristics, including stage, histology, grade and serum tumor markers, in the present study. Additionally, it was also revealed that serum levels of fibronectin were not associated with survival. Therefore, in the present study, fibronectin levels in serum were not a useful prognostic marker to predict tumor prognosis in patients with gastric cancer. Additionally, the link between serum fibronectin concentrations and chemosensitivity has raised the possibility of using fibronectin as a predictor of response to chemotherapy in patients scheduled to undergo various chemotherapeutic regimens. It was demonstrated in the present study that serum fibronectin levels may not be a potential predictor of clinical response to chemotherapy in gastric cancer patients. A contrasting finding was also observed in patients with other tumor types (5).

In conclusion, the present study revealed that serum levels of fibronectin were only a diagnostic marker in gastric cancer patients. However, its predictive and prognostic values were not determined in these patients. The small sample size and short follow-up time of the present study may be considered as significant limitation and may have influenced these results. However, the present study contributed to the literature, as it contained all stages and groups of disease. Further studies in a larger patient population are necessary to determine the potential clinical significance of this assay in patients with gastric cancer.

\section{References}

1. Pankov R and Yamada KM: Fibronectin at a glance. J Cell Sci 115: 3861-3863, 2002.

2. Hynes RO (ed): Fibronectins. 1st Edition. Springer-Verlag, New York, NY, 1989.

3. Williams CM, Engler AJ, Slone RD, Galante LL and Schwarzbauer JE: Fibronectin expression modulates mammary epithelial cell proliferation during acinar differentiation. Cancer Res 68: 3185-3192, 2008. 
4. Han S, Khuri FR and Roman J: Fibronectin stimulates non-small cell lung carcinoma cell growth through activation of Akt/mammalian target of rapamycin/S6 kinase and inactivation of LKB1/AMP-activated protein kinase signal pathways. Cancer Res 66: 315-323, 2006.

5. Jerhammar F, Ceder R, Garvin S, Grénman R, Grafström RC and Roberg K: Fibronectin 1 is a potential biomarker for radioresistance in head and neck squamous cell carcinoma. Cancer Biol Ther 10: 1244-1251, 2010.

6. David L, Nesland JM, Holm R and Sobrinho-Simoes M Expression of laminin, collagen IV, fibronectin and type IV collagenase in gastric carcinoma. Cancer 73: 518-527, 1994.
7. Grigioni WF, Biagini G, Errico AD, Milani M, Villanaci V, Garbisa S, Mattioli S, Gozzetti G and Mancini AM: Behavior of basement membrane antigens in gastric and colorectal cancer. Immunohistochemical study. Acta Pathol Jpn 36: 173-184, 1986.

8. Li Y, Chen Y, Tao Y, Wang Y, Chen Y and Xu W: Fibronectin increases RhoA activity through inhibition of PKA in the human gastric cancer cell line SGC-7901. Mol Med Rep 4: 65-69, 2011.

9. Eisenhauer EA, Therasse P, Bogaerts J, Schwartz LH, Sargent D, Ford R, Dancey J, Arbuck S, Gwyther S, Mooney M, Rubinstein L, Shankar L, Dodd L, Kaplan R, Lacombe D and Verweij J. New response evaluation criteria in solid tumours: revised RECIST guideline (version 1.1). Eur J Cancer 45: 228-247, 2009. 